Superalloys 2012: 12 ${ }^{\text {th }}$ International Symposium on Superalloys

\title{
THE VARIABILITY OF FATIGUE IN NOTCHED BARS OF IN100
}

\author{
Andrew H. Rosenberger ${ }^{1}$, Dennis J. Buchanan², D’Anthony A. Ward ${ }^{3}$, and Sushant K. Jha ${ }^{4}$ \\ ${ }^{1}$ Air Force Research Laboratory, Materials \& Manufacturing Directorate, AFRL/RXLMN, 2230 Tenth St, \\ Wright-Patterson Air Force Base, OH 45433-7817, USA \\ ${ }^{2}$ University of Dayton Research Institute, 300 College Park, Dayton, OH 45469, USA \\ ${ }^{3}$ Southern Ohio Council of Higher Education, Dayton OH, 45420, USA \\ ${ }^{4}$ Universal Technology Corporation, Dayton, OH, 45432, USA
}

Keywords: Notch Fatigue, IN100, Small Crack Growth

\begin{abstract}
IN100 is one of the workhorse nickel-base superalloys of the current fleet of turbine engines owned and operated by the US Air Force. Many of the life-limited locations on fracture critical IN100 components are associated with locations of stress concentrations, e.g. bolt holes, fillets, balance flange scallops, etc. The prior work by Jha, Caton, and others [1-3] examining the fatigue variability of nickel-base superalloys found a link between the minimum fatigue life and fracture mechanics. That is, the minimum fatigue life was predicted assuming immediate crack initiation and growth of a small crack. This prior work only examined the fatigue behavior of smooth fatigue bars. The current effort determined that the link between minimum fatigue life and fracture mechanics is applicable even when only a small volume of the material at the root of a notch is subject to the high stress conditions.
\end{abstract}

\section{Introduction}

Most fracture critical locations in structural components are associated with stress concentrations, bolt holes, fillets, broach slots, etc. There has been considerable work to assess the likelihood of fatigue failure at these locations since the early work of Neuber [4]. It is clear that the stress-strain response of the notch drives the crack initiation and growth and this is often treated in an empirical manner [5]. After a small crack is initiated, the growth of the crack is affected by the notch plasticity and changes in the crack closure [6-8]. Recent work has considered the importance of small crack growth during the critical period between crack initiation and detection at an engineering size [810]. However, all of these approaches consider crack initiation and growth as two distinct, damaging processes that occur at the notch location.

The present work is based on the fundamental hypothesis that the minimum fatigue life is based on the initiation of a fatal crack on the first cycle and that the entire fatigue life is spent propagating that crack to a critical size. This hypothesis has been repeatedly demonstrated by Jha, Caton and others, [1-3]. The important steps are to: 1) determine the mechanism of crack initiation and determine the distribution of initiation sizes, 2) measure [11] or model [12] the growth rate of the small crack that grows from the initiation size to a size large enough where linear elastic fracture mechanics (LEFM) is applicable, and 3) use LEFM to grow the crack to a critical size - failure. This hypothesis has been shown to hold for a number of materials at various test temperatures, but only under the conditions of a uniform stress, $\mathrm{Kt}=1$, and simple fatigue cycling.

The demonstration of the linkage between the minimum fatigue life, the usable life, and fracture mechanics means that life management philosophies can be simplified [13]. Furthermore, the development of new materials or the application of existing materials to new applications can be realized with a reduced fatigue data set as minimum fatigue allowables could be calculated using the three steps outlined above with minimal test data.

This paper goes through the steps to assess the minimum fatigue life for a notched test bar and verifies the prediction with sufficient test data to validate the approach.

\section{Material and Experimental Procedures}

The material is powder metal supersolvus heat treated IN100 that is identical to the material in [2]. The sources of crack initiation and their sizes have been well characterized in this material making it an ideal material to assess the influence of stress concentrations on the fatigue variability. The sources of crack initiation are predominately clusters of pores and nonmetallic particles, which lead to the minimum fatigue life [1]. Therefore, the distributions of these material characteristics from Reference [2] can be used directly in this study.

Double edge notch specimens having an elastic Kt of 1.89 were subject to fatigue at a stress ratio of 0.05 , temperature of $650^{\circ} \mathrm{C}$, and 20 cycles per minute $(0.33 \mathrm{~Hz})$. Figure 1 shows the geometry of the specimen. Button-head grip ends were used to ensure accurate alignment thereby eliminating the influence of the mechanical test system on the fatigue variability. Specimens were heated to the test temperature of $650^{\circ} \mathrm{C}$ using a resistance furnace controlled by thermocouples welded to the shoulder of the specimen. The temperature at the notch of the sample was correlated to that on the shoulder using an instrumented dummy. The initiation sites were identified and measured on the fractured specimens using SEM. Several small crack tests were conducted having nominally 40 x $20 \mu \mathrm{m}$ 3-D micro-notches machined using focused ion beam (FIB) in the geometric center of the two notches. These micro-notches act as crack starters to enable the monitoring of the small crack growth rate using cellulose acetate replication. In these tests, the cycling was interrupted at a predetermined cycle, the load was ramped to the minimum level, and power to the furnace was cut. The specimen cooled to room temperature in approximately 1 hour and the replication was conducted with the specimen at $75 \%$ of the maximum load to open the crack(s). The replicas were mounted on glass slides and the crack lengths were identified and measured using an optical microscope.

The tests were conducted with a maximum applied net section stress of 800 and $900 \mathrm{MPa}$ with the intent of maintaining the same fatigue life range as the $\mathrm{Kt}=1$ tests from [2]. Loading to these levels resulted in elastic stresses at the notch tip 
substantially higher than yield in the first cycle, thereby necessitating an elastic-plastic stress analysis of the specimen.
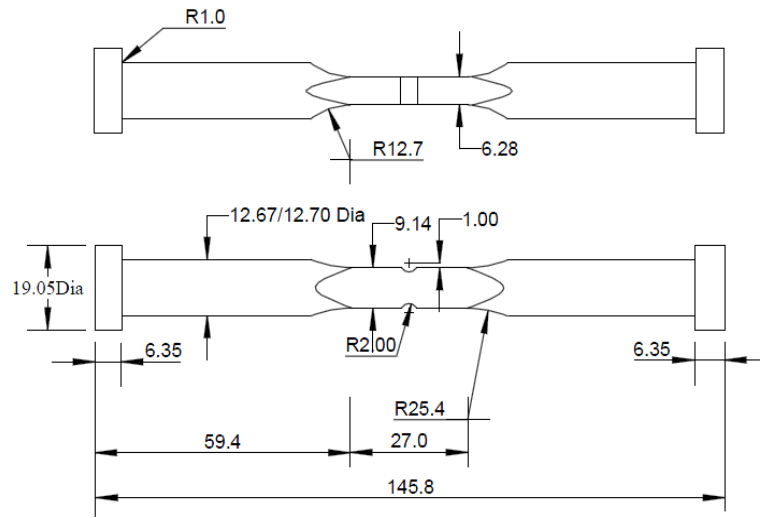

Figure 1. Double edge notch specimen, (dimensions in mm).

\section{Results and Discussion}

Notch Stress Analysis

Accurate modeling of the stresses on the plane of the crack in the notch is critical to accurately calculate the stress intensity factor for the growing cracks. A one-eighth model of the notch specimen was developed in ABAQUS having refined mesh spacing at the notch root of $50 \mu \mathrm{m}$. The plasticity model was a nonlinear mixed isotropic-kinematic hardening law based on the stabilized cyclic strain response. The model parameters were determined using the ABAQUS fitting procedure and strain controlled, stabilized hysteresis loops from fully reversed, $\mathrm{R}_{\varepsilon}=-1$, and fully tensile, $R_{\varepsilon}=0$, experiments. The ABAQUS model resulted in stabilized hysteresis loops after $\sim 8$ cycles. Figure 2 shows the axial stress field at the notch root for a net section stress of $800 \mathrm{MPa}$ after 8 loading cycles. Since the material at the surface of the notch yields, the maximum stress is slightly subsurface and drives deeper with an increase in the maximum net section stress.

\section{$\underline{\text { Stress Intensity Factor Estimation }}$}

The stress intensity factor was estimated for cracks growing in the notch using AFGROW [14]. First, the elastic stress field, figure 3, from an ABAQUS run was used to determine the applied maximum and minimum $\mathrm{K}$ during the cycle - consistent with the requirements for linear elastic fracture mechanics. The plasticity was incorporated by calculating a static $\mathrm{K}$ from the residual stress after plastic cycling, figure 4. This residual stress was incorporated in AFGROW as a superposition of a mean stress and the cyclic stress. Different residual stress profiles were used for the two stress levels as the depth and level of plasticity was dependant on the load level as shown in figure 5. It should be noted that the low stress grind (LSG) machining residual stress profile was measured on a representative specimen using x-ray diffraction, figure 5 , and was found to be shallow compared to the plasticity induced residual stress and hence was not used in estimating the stress intensity factor. Also shown in that figure is the initiation size distribution which tends to be as deep as the machining residual stress.

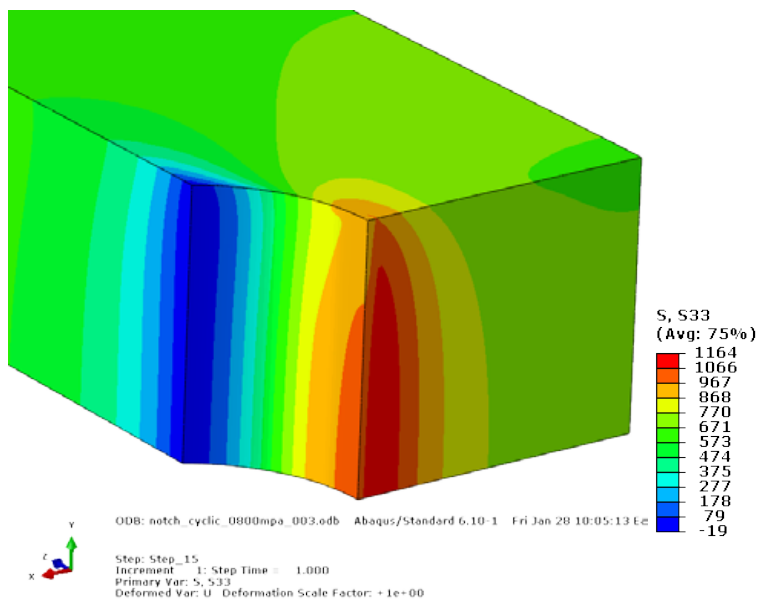

Figure 2. Axial stress at the notch for a net section stress of 800 $\mathrm{MPa}-\mathrm{a}$ one-eighth model was used.

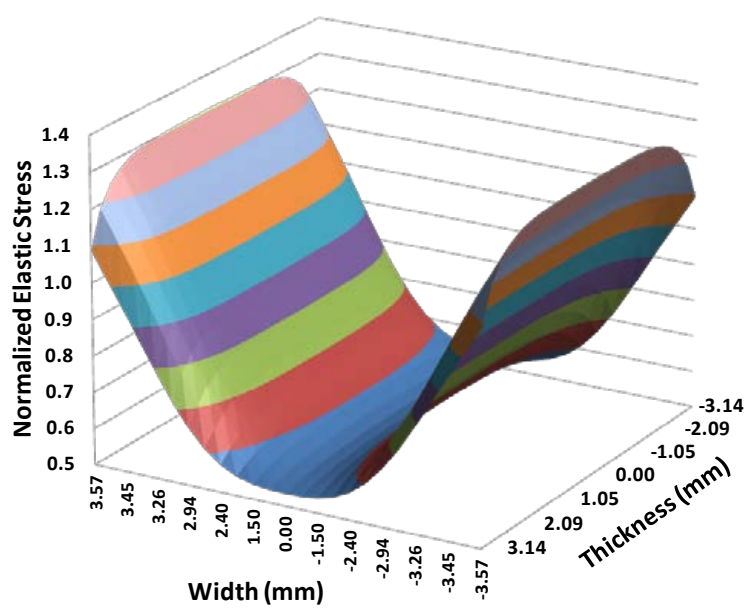

Figure 3. Normalized elastic stress distribution on the minimum section notch plane ( $1=$ net section stress).

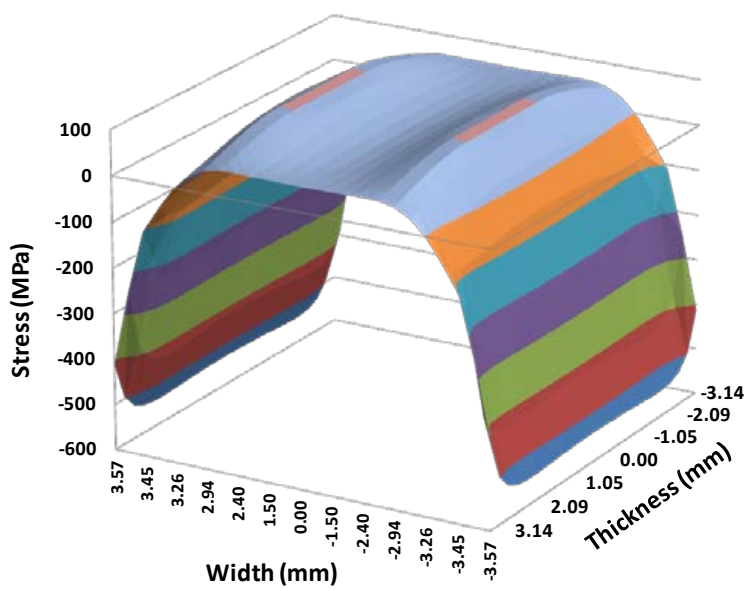

Figure 4. Stabilized stress at minimum load from elastic-plastic ABAQUS analysis for a maximum net section stress of $800 \mathrm{MPa}$. 


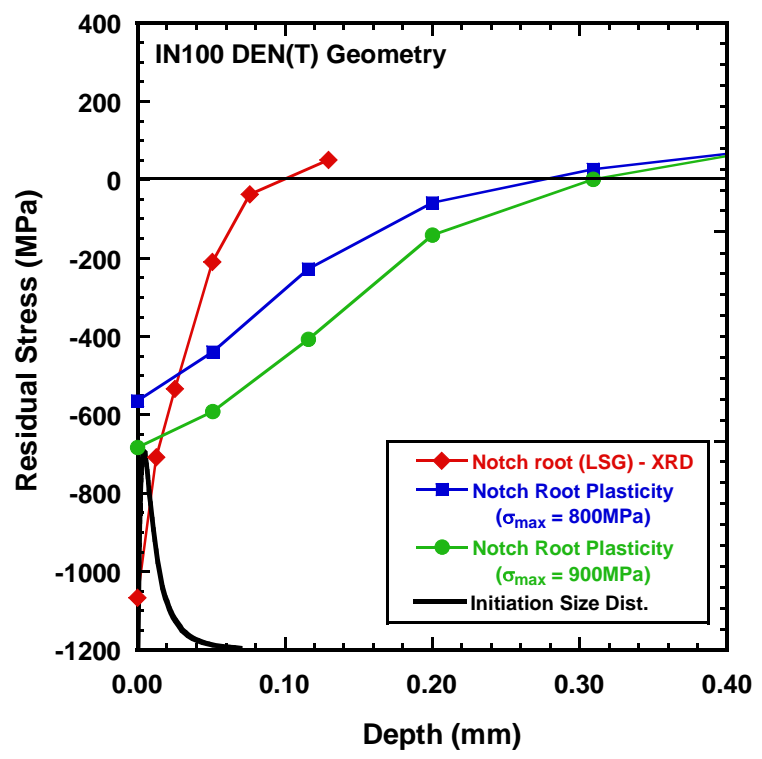

Figure 5. Machining residual stress profile and notch plasticity residuals as a function of depth from the notch surface.

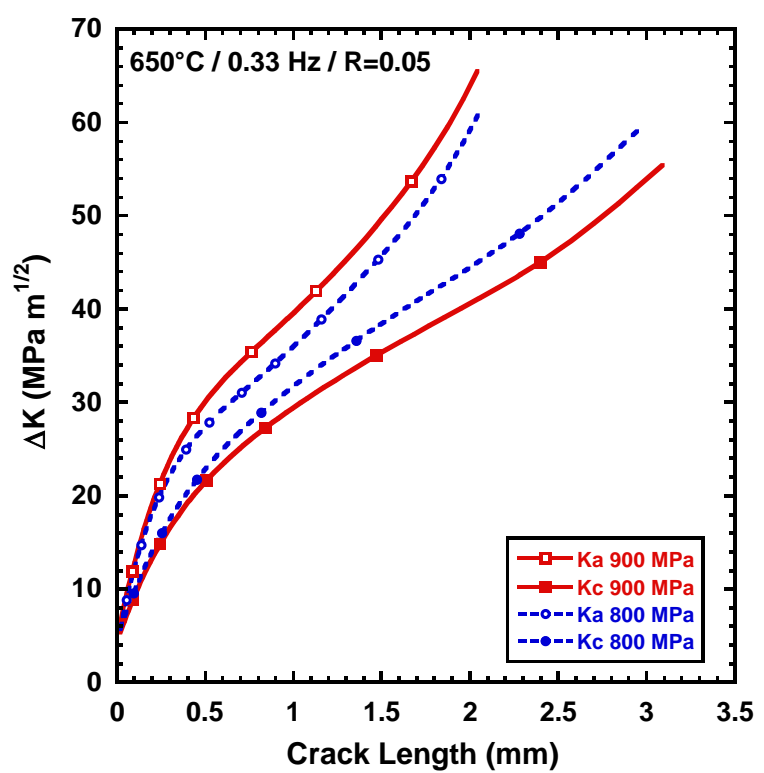

Figure 6. Stress intensity factor range, $\Delta \mathrm{K}$ versus crack depth and half surface length for specimens subject to maximum applied stresses of 800 and $900 \mathrm{MPa}$.

The stress intensity factor was determined using AFGROW and a simple surface crack in a rectangular bar that matched the net section of the notched samples. The notch stress gradient, figure 3 , was introduced as the cyclic stress and the plasticity induced residual stress, figure 4 , were superposed to produce an effective stress intensity. AFGROW was run with a simple Paris Law material model as the goal was to determine the stress intensity factor not the crack growth rate. Figure 6 shows the stress intensity factor range as a function of crack length for the two stress levels and the K's on the surface, c, and the depth, a, directions. Since there is a significant stress gradient, the crack will tend to grow with an aspect ratio, a/c of less than 1 . These K's were used for the subsequent data analysis and crack growth predictions.

\section{$\underline{\text { Fatigue Variability }}$}

Figure 7 shows the cumulative distribution of fatigue life for notched test bars compared to smooth test bars from reference [2]. The maximum net section stress levels of 800 and $900 \mathrm{MPa}$ produce mean fatigue lives similar to smooth bars tested at 1000 and $1100 \mathrm{MPa}$ indicating that notch strengthening is taking place, as expected. The bulk of the notch testing was conducted at a maximum stress of $800 \mathrm{MPa}$ to produce lives that are more representative of structural components. Overall, it is apparent that the variation in life is similar for the two test geometries. In fact, the variability in life is greater for the notch specimens tested at a maximum stress of $800 \mathrm{MPa}$ which may be a result of the vastly different interrogation volumes for the two geometries. The notch specimens have a high stress volume no larger than 20 $\mathrm{mm}^{3}$ while the smooth bars are interrogating over $280 \mathrm{~mm}^{3}$ each. Even if only the outer skin $(0.5 \mathrm{~mm})$ of the smooth test bars is considered the critical failure location (due to environmentally enhanced crack growth), the volume would be $\sim 130 \mathrm{~mm}^{3}$. Since the initiation sites for crack initiation in this material under these loading conditions are predominately clusters of pores and nonmetallic particles [2], the probability of having a large initiation source in the highly stressed volume will not be as high as for the smooth geometry. This would result in some notch specimens having a longer life than smooth bars. However, if a large initiation source is in the highly stressed volume, then the life would be much shorter in the notched specimen than that in a smooth bar. Hence the increase in scatter seems to make physical sense.

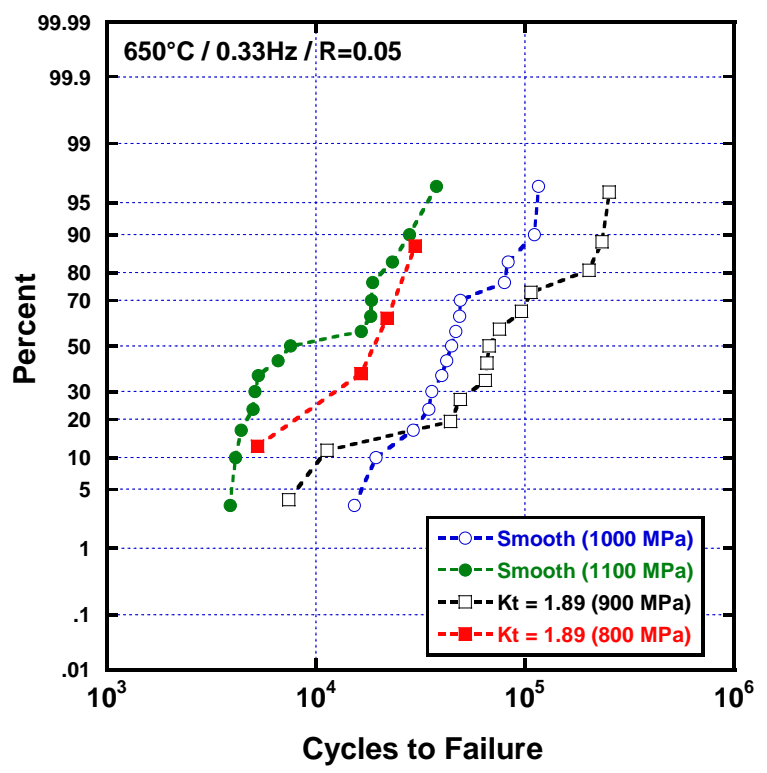

Figure 7. Cumulative distribution function of fatigue life for specimens having $\mathrm{Kt}=1$ [2] and 1.89 . 
Examination of the fracture surfaces of the failed specimens in the SEM found that typically a single crack initiated and grew to failure from one of the notches. The source of crack initiation was found to be a nonmetallic particle or cluster of trapped gas pores that are common in powder metallurgy materials. The majority of the initiation sites were near the surface of the notch. The maximum depth of the initiation site was $557 \mu \mathrm{m}$ for a specimen that failed from a nonmetallic particle. Only nonmetallic particles were found at deep initiation sites indicating that they are rare relative to the gas pores and also, that they must have a tensile residual stress around them, CTE induced, that aids in their crack initiation potency. A comparison of the initiation source and its size (equivalent circle diameter) is shown in figure 8 with the results of smooth specimens [2].

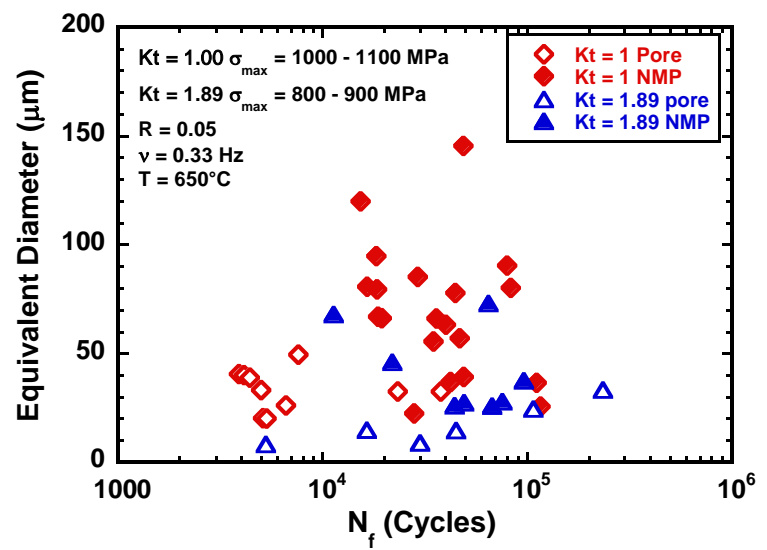

Figure 8. Crack initiation source and size versus number of cycles to failure for smooth [2] and notched specimens.

In both cases, the largest initiation site does not necessarily result in the shortest fatigue life. However the notched specimens had generally smaller initiation sizes than the smooth bars. This must be due to the greater potency of a smaller defect near the root of the notch. The stress intensity factor would be substantially greater there resulting in faster growth even at smaller crack sizes.

\section{Small Crack Growth}

The key to enable the prediction of the minimum fatigue life is understanding the growth rate of the small crack as it extends beyond the initiation site. To this end several tests were conducted with artificial FIB crack starters in the notch and replicated to measure the small crack growth rates. This also allows a comparison of the small crack growth rate for cracks in the $\mathrm{Kt}=$ 1.89 stress field to cracks growing in a uniform stress field [2]. Cracks initiated readily at the crack starter but due to the concentrated stress some cracks also initiate naturally. Figure 9 shows the crack growth rate for the artificially and naturally initiated small cracks growing in the $\mathrm{Kt}=1.89$ specimens along with conventional long and small crack growth rate data from [2]. (The growth rates and K's are for surface cracks.) It can be seen that overall, the small crack growth rates for the two conditions are similar with the $\mathrm{Kt}=1.89$ growing slightly faster and demonstrating slightly more variability in crack growth rate. The slight increase in growth rate is likely due to the different stress conditions for the small cracks. The notch specimens have a global stress ratio of 0.05 but have a local stress ratio in the notch that is location specific but on the order of -0.4 to -0.5 . This negative stress ratio could contribute to the growth rate. Small cracks tend to have low levels of crack closure. But small cracks growing in a notch stress field are predicted to have higher levels of crack closure [6]. However, careful measurements of the closure levels of small cracks growing in strain controlled specimens demonstrate that the opening load is low and can, in fact, be negative [15]. The lowest initial closure (negative) was found in the 0 to maximum strain cycle where the first cycle yield resulted in a compressive minimum stress. This is similar to the first cycle yield in the present notch specimens.

Another source of faster growth rates in the notch specimen is the merging of a primary crack with a secondary crack resulting in a jump in the crack length. Figure 10 shows snapshots of a growing, naturally initiated crack. In front of the initial small crack, arrow, a larger crack initiates and grows faster than the original crack. Note that the maximum stress is slightly sub surface, so a larger initiation source may be located just below the surface to initiate the second crack. This growth process will also lead to the greater variability in crack growth rate for the notch specimens observed in figure 9. Additionally the data analysis technique will increase the variability of the notch crack growth rates. The $\mathrm{da} / \mathrm{dN}$ for the notch specimens was calculated using a simple secant method while the smooth bar data was regressed using a sliding 3 or 5 point polynomial.

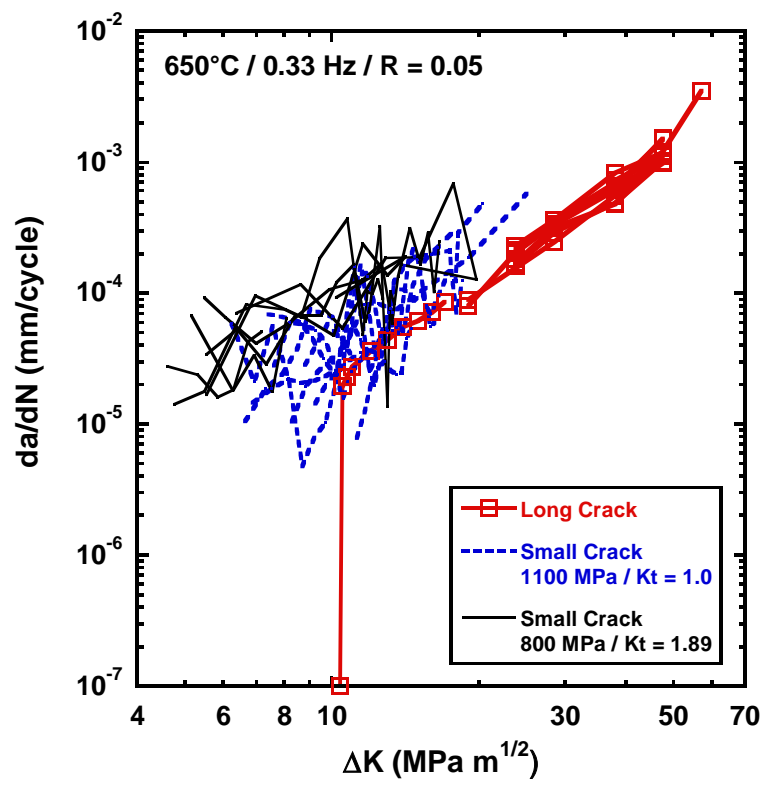

Figure 9. Crack growth rate versus stress intensity factor for long cracks, small cracks growing in a uniform stress field, $\mathrm{Kt}=1$ [2] and cracks growing in the notch stress field, $\mathrm{Kt}=1.89$.

The similarity in small crack growth behavior between smooth and notched specimens is encouraging but also highlights that model approaches are needed to predict the stress ratio effects in small cracks like those in use for long crack fatigue [16]. Small crack growth also has greater inherent variability and methods are needed to predict this variability for use in probabilistic models. There are interesting model approaches that are being developed [12] and more work in this area could be fruitful. 

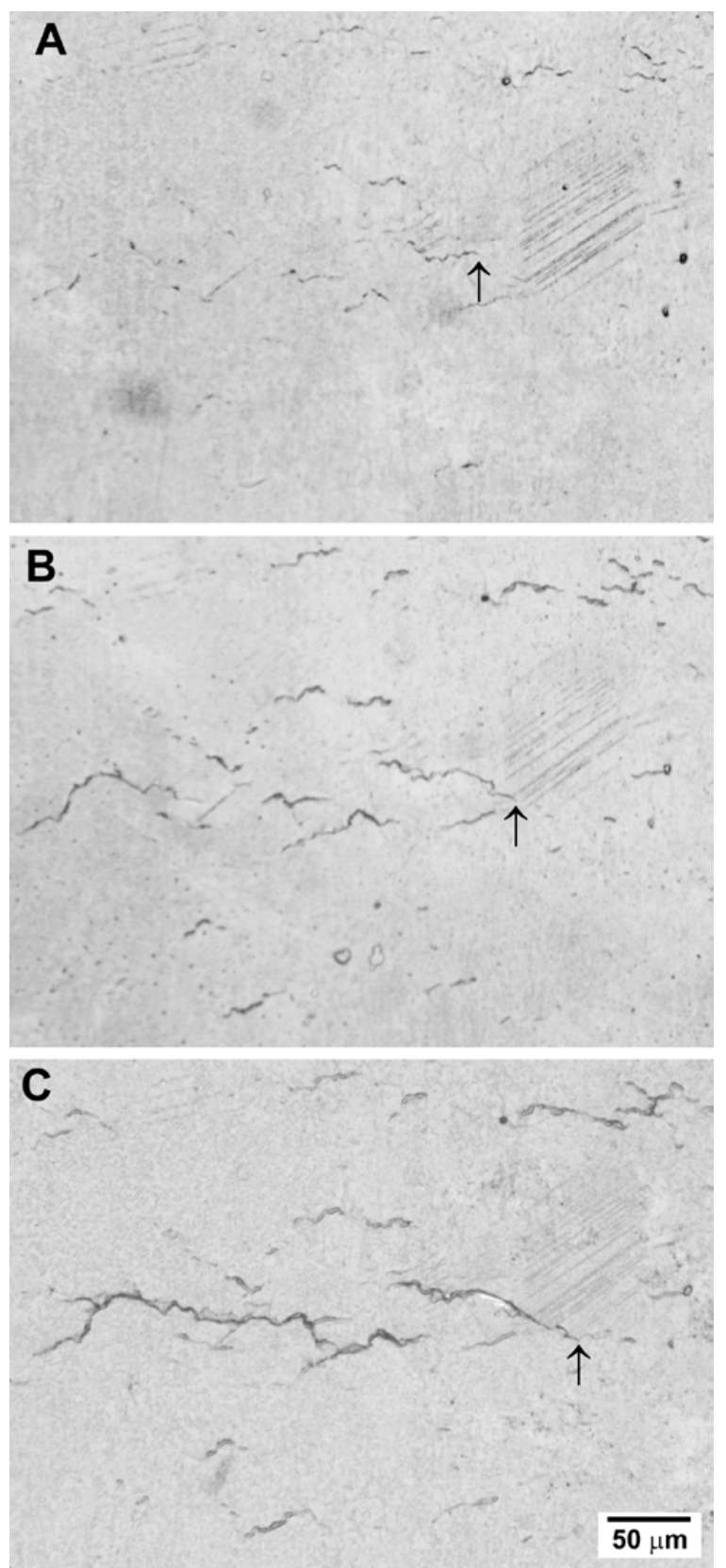

Figure 10 Replica images showing the variability in small crack growth for a specimen tested at $650^{\circ} \mathrm{C} / 0.33 \mathrm{~Hz} / \mathrm{R}=0.05 / \sigma_{\max }=$ $800 \mathrm{MPa} / \mathrm{Kt}=1.89$. a) 550 cycles, b) 1550 Cycles, c) 2250 cycles. Dominant crack in a) marked with $\uparrow$.

\section{$\underline{\text { Probabilistic Minimum Fatigue Life Prediction }}$}

The minimum fatigue life for the notched bars was simulated using the Monte Carlo (MC) method [1, 17]. The distributions of crack initiation size and the variation in small crack growth Paris Law parameters, $C$ and $m$ were used. Briefly, the small crack growth data were fit to the Paris Law and the resultant distributions of $C$ and $m$ values were placed in a joint probability distribution for Monte Carlo sampling. The initial crack size was modeled as a lognormal distribution [17] of only the nonmetallic particles since these were the largest initiation sources. The $\mathrm{K}$ versus crack length relation, figure 6 , was used to update the stress intensity factor of the simulation. The cracks were then grown to failure. The methodology is described in more detail in References 1 and 17 . The cumulative distribution function of life from 10,000 MC runs using the smooth bar small crack growth rates from reference 2 is shown in figure 11 . The $1 / 1000$ life (B0.1) for the two applied stresses are 7200 cycles for a net section stress of $800 \mathrm{MPa}$ and 5790 cycles for a net section stress of $900 \mathrm{MPa}$. These compare reasonably well with the minimum observed lives of 7414 and 5262 cycles, respectively. However, these values are slightly anti conservative especially considering the limited number of tests conducted and the relatively small volume of material (and initiation sites) interrogated.

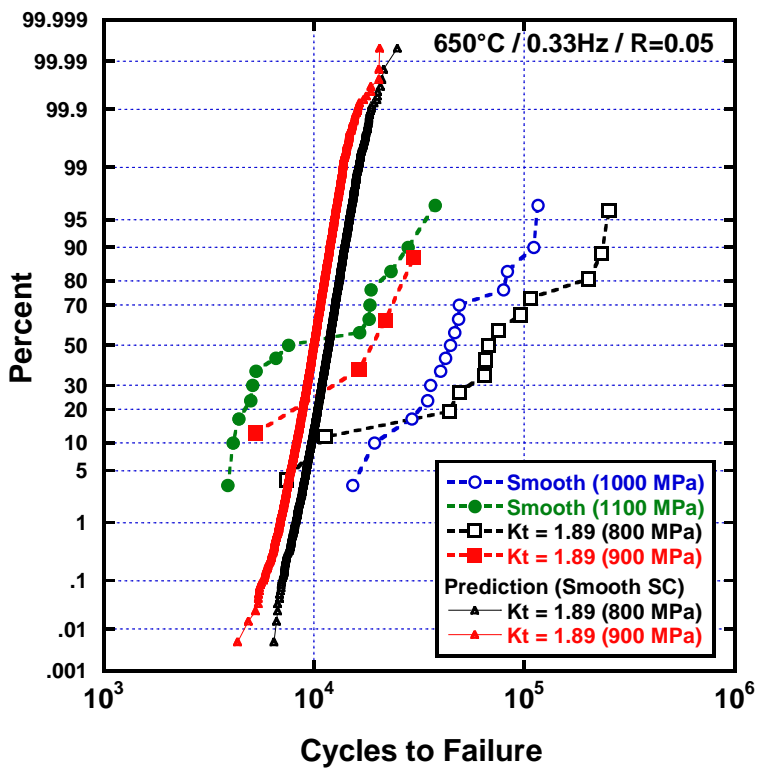

Figure 11. Probabilistic prediction of minimum fatigue life for $\mathrm{Kt}=1.89$ using smooth bar small crack growth rates.

A second set of 10,000 MC runs was conducted using the small crack growth rates as measured on the notch test bars, shown in Figure 12. The predicted distributions of minimum fatigue lifetime for the notched specimens are in better agreement with experimentally observed minimum lifetimes. The predicted B0.1 lifetimes were 4430 and 3750 cycles compared to the previously mentioned minimum observed lives of 7414 and 5262 cycles at 800 and 900 MPa net section stress, respectively. These predictions use the same initiation distribution of nonmetallic particles seen in a greater number of smooth specimens, Figure 8, in order to account for the full distribution in crack initiation size if more volume was sampled. However, since the present experiments have smaller crack initiation sizes, the predictions should be and are conservative with respect to these particular experimental points.

These probabilistic calculations demonstrate that the method based on fracture mechanics is promising in terms of capturing the minimum fatigue life population even in the case of notched specimens. 


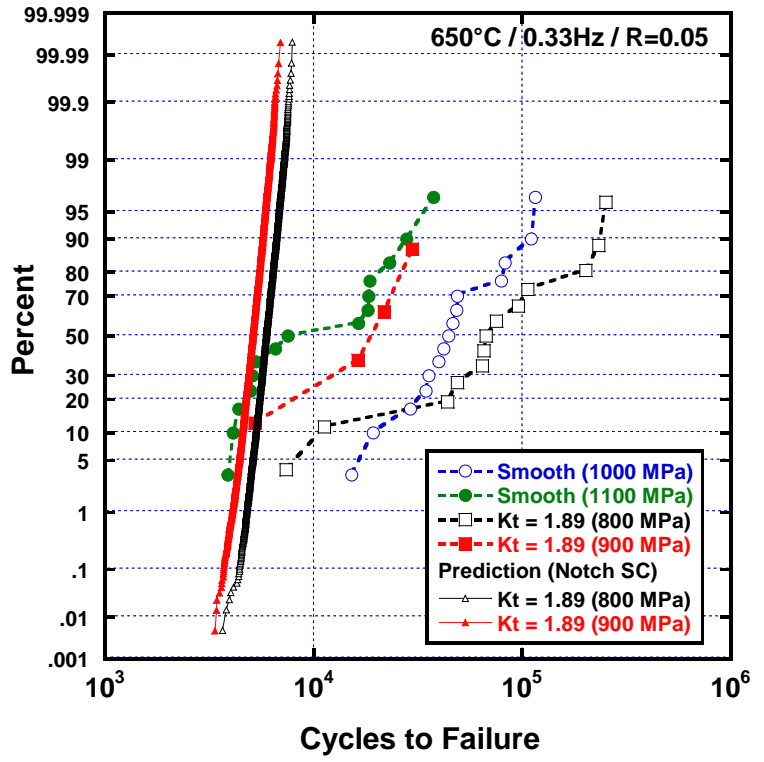

Figure 12. Probabilistic prediction of minimum fatigue life for $\mathrm{Kt}=1.89$ using notched bar small crack growth rates.

\section{Conclusions}

The variability of fatigue at $650^{\circ} \mathrm{C}$ in identical notch test bars of powder metallurgy IN100 was studied and the minimum fatigue life was accurately predicted using fracture mechanics. The following general conclusions were found in this study.

- The mechanisms of crack initiation are the same in smooth and notched test bars for the conditions tested.

- The small crack growth rates in smooth bars are slightly lower than the rates for cracks growing in notched bars.

- The minimum fatigue life is accurately predicted using the fracture mechanics provided that the actual small crack growth rates are incorporated.

These results also indicate that the small interrogated volume in notch specimens is sufficient to express the scatter in notch fatigue using only a handful of specimens. Furthermore, if the small number of notch bars captures the minimum fatigue life, then a turbine engine disk having a number of life limiting, stress concentrations should find a minimum fatigue condition in each component. This further indicates that the US Air Force is currently operating a redundant lifing philosophy [13] wherein both safe life and damage tolerance are concomitantly required for all locations in fracture critical components. A single lifing requirement incorporating this probabilistic fracture mechanics approach may lead to improved component life utilization and a reduction in the data requirements for new materials or systems.

\section{Acknowledgements}

This work was performed at the Air Force Research Laboratory, Materials and Manufacturing Directorate, Wright-Patterson Air Force Base, OH. The partial financial support from the USAF FY10 Section 219 Venture Fund is gratefully acknowledged. SKJ acknowledges essential support provided by the Air Force Office of Scientific Research (AFOSR) LRIR Task 11RX01COR with
Dr. David Stargel as the program manager. The authors also wish to acknowledge Dr. Reji John, Dr. Robert Brockman and Mr. Peter Phillips for their helpful technical discussions and assistance with the stress intensity factor calculations and ABAQUS modeling.

\section{References}

1) S.K. Jha, M.J. Caton, and J.M. Larsen, "Mean vs. LifeLimiting Fatigue Behavior of a Nickel-Based Superalloy, Superalloys 2008, Eds. R.C. Reed etc, TMS, pp. 565-572, 2008.

2) S. K. Jha, M. J. Caton, and J. M. Larsen, "Effect of Grain Size on the Small-Crack Growth Behavior in a Ni-Base Superalloy under Dwell Conditions" presented at Propulsion Safety and Affordable Readiness conference, Jacksonville, FL, May 2011.

3) M.L. Brogdon and A.H. Rosenberger, "Evaluation of the Influence of Grain Structure in the Fatigue Variability in Waspaloy,” Superalloys 2008, Eds. R.C. Reed etc, TMS, pp. 583-588, 2008.

4) H. Neuber, "Theory of Stress Concentration for ShearStrained Prismatical Bodies with Arbitrary Nonlinear StressStrain Law,” Trans ASME J. Appl. Mech., 8, 1961, pp. 544550.

5) G. Härkegård and G. Halleraker, "Assessment of the Methods for Prediction of North and Size Effects at the Fatigue Limit Based on Test Data by Böhm and Magin,” IJF, 32, 2010, pp. 1701-1709.

6) T. Ye, H. Jiawen, and H. Naisai, "Effect of Notch Stress Field and Crack Closure on Short Fatigue Crack Growth," Fat. Fract. Eng. Mater. Struct., 13(4) 1990, pp. 423-430.

7) D.L Ball, "A Model for the Inclusion of Notch Plasticity Effects in Fatigue Crack Growth Analysis,” J. ASTM Int., 1(9) 2004, Paper ID JAI19045.

8) M. Aratani and J.F. Knott, "The Growth of Short Fatigue Cracks Ahead of a Notch in High Strength Steel,” Eng. Failure. Anal., 17, 2010, pp. 200-207.

9) N. Ranganathan, et al., "Fatigue Crack Initiation at a Notch," IJF, 33, 2011, pp. 492-499.

10) D. Taylor, "A Mechanistic Approach to Critical-Distance Methods in Notch Fatigue,” Fat. Fract. Eng. Mater. Struct., 24, 2001, pp. 215-224.

11) Guidelines for Measuring the Growth Rates of Small Fatigue Cracks, ASTM Standard E-674 Annex 3, ASTM International, 2011.

12) I. Simoinovski and L. Cizelj, "The influence of the Grain Structure on Microstructurally Short Crack," J. Eng. for Gas Turb. \& Pwr. 131, 2009, pp. 042903-1,8.

13) Engine Structural Integrity Program, Department of Defense Handbook, MIL-HDBK-1783B, 15 February 2002.

14) http://afgrow.net/ accessed 13 February 2012.

15) A.H. Rosenberger and H. Ghonem, "Effect of Cycle Mean Strain on Small Crack Growth in Alloy 718 at Elevated Temperatures," Fat. Fract. Eng. Mater. Struct., 11(15) 1992, pp. 1125-1139.

16) K. Walker, "The Effect of Stress Ratio during Crack Propagation and Fatigue for 2024-T3, STP 462, ASTM, 1970, pp. 1-14.

17) S.K. Jha, M.J. Caton, and J.M. Larsen, “A new paradigm of fatigue variability behavior and implications for life prediction,” Mat. Sci Eng. A 468-470, 2007, pp.23-32. 